\title{
Heavy Drinker
}

National Cancer Institute

\section{Source}

National Cancer Institute. Heavy Drinker. NCI Thesaurus. Code C126384.

A man who drinks more than 14 standard drinks per week or a woman who drinks more

than 7 standard drinks per week. 